\title{
Integrierte Versorgung psychisch Kranker Erfahrungen im Netzwerk
}

\author{
Siegfried Stephan \\ Facharzt für Psychiatrie und Psychotherapie, Facharzt für Psychosomatische Medizin und Psychotherapie, \\ Psychoanalyse und spezielle Schmerztherapie, Mainz \\ Vorsitzender des Netzes für Seelische Gesundheit Mainz e. V.
}

Eine weltweite Studie der Weltgesundheitsorganisation (WHO) aus dem Jahr 2001 zeigte bei den 10 häufigsten Ursachen für die mit Beeinträchtigung gelebten Lebensjahre in den Industrieländern folgende Ergebnisse: Die Depression lag mit 8,39 Millionen Lebensjahren weltweit ganz vorne auf Platz 1 der Rangliste. Die Deutsche Angestellten Krankenkasse (DAK) hat erhoben, wie oft und warum ihre Mitglieder in den Jahren von 1997-2001 krankgeschrieben waren: Während die gesamtdurchschnittlichen Krankheitstage um $16 \%$ anstiegen, konnte eine Zunahme an Krankheitstagen aufgrund psychischer Störungen von $\mathbf{5 1} \%$ in demselben Zeitraum verzeichnet werden. Bereits jetzt werden 6-12\% des Bruttosozialprodukts in den entwickelten Ländern für die Gesundheitsfürsorge ausgegeben; von diesen fallen $4 \%$ auf die Kosten infolge von psychischen Störungen. Der überwiegende Anteil ist die direkte Konsequenz von inadäquat behandelten depressiven Störungen.

Patienten mit Depressionen suchen nur zu etwa einem Drittel überhaupt ärztliche Hilfe. Von diesen werden lediglich $50 \%$ als solche erkannt und von diesen erkannten Depressionen wird wiederum nur ein kleiner Teil adäquat behandelt. Häufig kommen Patienten erst mit schweren therapieresistenten und chronifizierten Depressionen zum Facharzt. Um jedoch einer Chronifizierung entgegenwirken zu können, muss das Ziel eine schnelle Diagnostik und eine adäquate Behandlung sein. Hierzu bedarf es einer engen Zusammenarbeit zwischen Hausärzten, Fachärzten und (teil-)stationären und komplementären Behandlungsmöglichkeiten.

Netz für seelische Gesundheit Mainz e. V. Um dem Manko, das sich aus einer unzureichenden Zusammenarbeit ergibt, entgegenzuwirken, gründeten niedergelassene Psychiater und Psychotherapeuten zusammen mit einigen Hausärzten und dem Deutschen Paritätischen Wohlfahrtsverband (DPWV) als Träger einer psychiatrischen Tagesklinik und psychiatrischen Institutsambulanz 2001 in Mainz das Netz für seelische Gesundheit Mainz e.V. (NsG Mainz e.V.). Die Netzmitgliederzahl stieg innerhalb von fünfein- halb Jahren von 12 auf 44 und hat seitdem die Behandlungsmöglichkeit von psychisch und psychosomatisch Erkrankten deutlich verbessert. Um eine weitere Integration der verschiedenen Versorgungsmöglichkeiten zu erreichen, nutzte das Netz für seelische Gesundheit die Möglichkeit der Integrierten Versorgung (IV), wie sie mittlerweile im SGB V § 140 beschrieben ist. Dadurch ist eine sektorenübergreifende Behandlung ermöglicht worden, wie dies vorher nicht der Fall war.

\section{Projekt ,Integrierte Versorgung“}

Im Frühjahr 2006 wurde ein Vertrag mit der Techniker Krankenkasse über eine Integrierte Versorgungsbehandlung von Patienten geschlossen (Abb. 1). Patienten, die ambulant nicht mehr adäquat behandelt werden können, wurden bisher in eine Klinik oder Tagesklinik eingewiesen. Nun können sie im Rahmen des Projekts weiter durch den niedergelassenen Arzt/Psychotherapeuten behandelt werden und zusätzlich die komplementären Möglichkeiten einer Institutsambulanz, Tagesklinik und sonstiger Leistungserbringer nutzen. Vorteil dieses Projekts ist es, dass die Möglichkeiten des „Krankenhauses ohne Krankenhaus“ genutzt werden können.

Wichtiges Ziel für das Netz für seelische Gesundheit Mainz e.V. war es von Anfang an, den Medizinischen Dienst der Krankenkassen Rheinland-Pfalz mit in das Projekt einzubeziehen, um möglichen Reibungsverlusten entgegenzuwirken. Eine Auswertung der Daten hat bereits begonnen, insbesondere in Bezug auf Veränderungen der Parameter Arbeitsunfähigkeitstage, stationäre Aufenthalte etc.

\section{Zur Projektbeschreibung}

Ein Patient ist nur dann für die Einschreibung in dieses Projekt geeignet, wenn aufgrund seiner Erkrankung die Erbringung mehrerer 


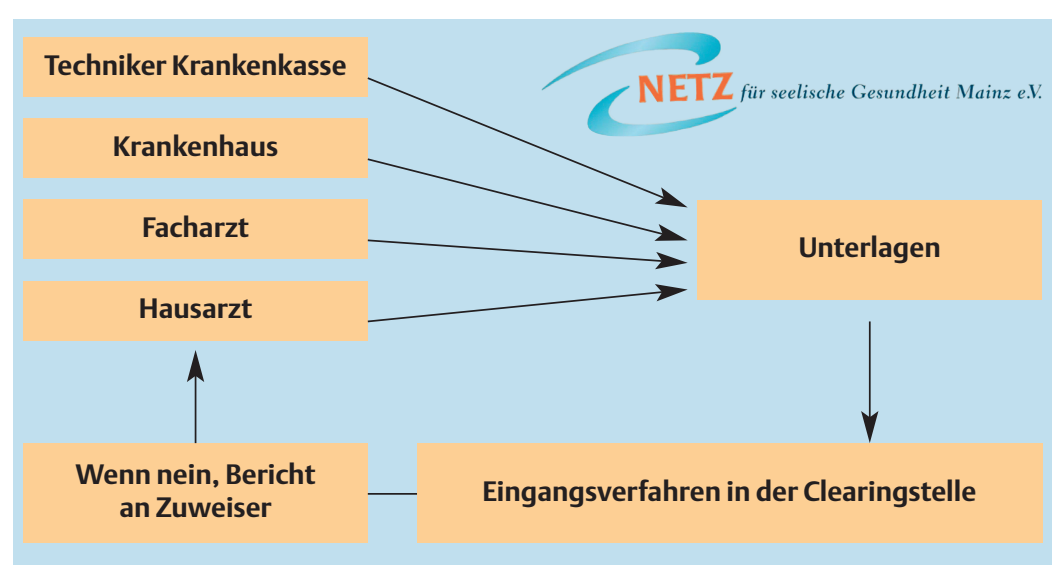

Abb. 1 Ambulante Krankenhausversorgung.

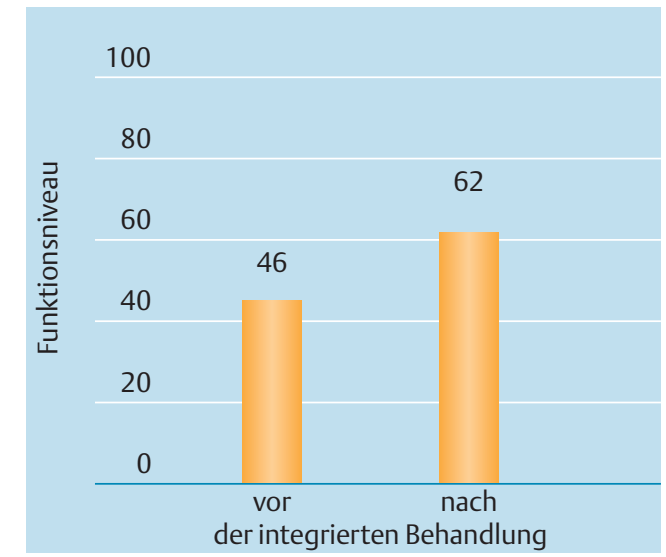

Abb. 2 Ergebnisse des GAF-Tests.
Leistungskomplexe nebeneinander erforderlich ist. Zudem soll im Team die auf den individuellen Bedarf an Komplexleistungen abgestellte multiprofessionelle Betreuung im Rahmen ambulanter Behandlung zur Verfügung gestellt werden.

Folgende Leistungskomplexe werden angeboten:

- ärztliche Behandlung

- psychotherapeutische Behandlung

- psychiatrische Pflege

- Ergotherapie

- Arbeitstherapie

- Soziotherapie

- Milieutherapie (von der tagesklinischen Betreuung bis hin zur Betreuung im häuslichen Umfeld)

- gemeinsame Visite aller Behandler mit anschließender Fallbesprechung.

Um eine qualifizierte Behandlung im IV-Projekt zu gewährleisten, wurden die folgenden Quali-

fikationen der Behandler definiert:

- Für niedergelassene Leistungserbringer werden u.a. festgehalten:

- Zulassung oder Ermächtigung

- Mitgliedschaft im NsG e.V.

- Qualitätsmanagement-Ausbildung nach ISO (NsG e.V. hat eigenes QM durchlaufen)

- regelmäßige Teilnahme an Fortbildungen inner- und außerhalb des Netzes.

- Niedergelassene ärztliche und psychologische Psychotherapeuten sowie Kinder- und Jugendpsychotherapeuten benötigen zusätzlich die erforderliche Fachkunde.

- Für die Tagesklinik und die psychiatrischen Institutsambulanzen (PIA) zusätzlich erforderlich sind die allgemein für Kliniken bzw. die PIA geltenden Voraussetzungen.

Im Projekt werden je nach notwendiger Behandlungsintensität die Patienten in sogenannte Hilfebedarfsgruppen von 1 bis 7 nach dem wöchentlichen Zeitbedarf eingeteilt. Die Koordination der Leistungen erfolgt durch einen federführenden Behandler. Fallbesprechungen finden regelmäßig statt. Die Vergütung erfolgt pauschalisiert und wird intern zwischen den am Projekt Beteiligten geregelt.

\section{Ergebnisse}

Für uns Behandler hat das Projekt Folgendes erbracht: Es gibt bei einer guten Koordination der Behandlung die Möglichkeit, die Patienten in Krisensituationen weiterzubehandeln. Damit kann punktuell eine größere Zufriedenheit mit der eigenen Arbeit trotz der schwierigen Situation des Gesundheitssystems erreicht werden.

Zur Evaluation der Patientenzufriedenheit werden zu Beginn, während und am Ende der Behandlung verschiedene Testungen durchgeführt, so z.B.

Tab. 1 Test zur punktuellen Messung der Patientenzufriedenheit: SCL-90-R. In den Spalten Beginn und Ende sind die Mittelwerte zu Beginn und Ende der Integrierten Versorgung aufgelistet, $\mathrm{d}=\left(\mathrm{MW}_{\text {Beginn }}-\mathrm{MW}_{\text {Ende }}\right) /$ Standardabweichung, gepoolt, $n=13$.

\begin{tabular}{|c|c|c|c|c|c|}
\hline SCL-90-R-Skala & $\mathrm{MW}_{\text {Beginn }}$ & $\mathbf{M W}_{\text {Ende }}$ & t-Wert & $\begin{array}{l}\text { Signifi- } \\
\text { kanz p }\end{array}$ & $\begin{array}{l}\text { Effekt- } \\
\text { stärke d }\end{array}$ \\
\hline Somatisierung & 57,9 & 48,9 & 4,19 & $<0,001$ & 1,2 \\
\hline Zwanghaftigkeit & 70,5 & 58,0 & 4,51 & $<0,001$ & 1,4 \\
\hline $\begin{array}{l}\text { Unsicherheit im } \\
\text { Sozialkontakt }\end{array}$ & 65,5 & 54,9 & 4,74 & $<0,001$ & 1,2 \\
\hline Depressivität & 68,8 & 55,9 & 4,30 & $<0,001$ & 1,4 \\
\hline Ängstlichkeit & 62,4 & 55,2 & 2,49 & $<0,05$ & 0,6 \\
\hline $\begin{array}{l}\text { Aggressivität/ } \\
\text { Feindseligkeit }\end{array}$ & 58,9 & 50,8 & 2,77 & $<0,05$ & 0,7 \\
\hline phobische Angst & 61,6 & 50,2 & 3,27 & $<0,01$ & 1,0 \\
\hline paranoides Denken & 59,5 & 50,2 & 3,14 & $<0,01$ & 1,1 \\
\hline Psychotizismus & 63,9 & 50,9 & 6,23 & $<0,001$ & 2,1 \\
\hline $\begin{array}{l}\text { Global Severity } \\
\text { Index (GSI) }\end{array}$ & 66,2 & 54,5 & 4,85 & $<0,001$ & 1,5 \\
\hline $\begin{array}{l}\text { Positive Symptom } \\
\text { Distress Index }\end{array}$ & 63,9 & 54,3 & 3,73 & $<0,01$ & 1,3 \\
\hline $\begin{array}{l}\text { Positive Symptom } \\
\text { Total }\end{array}$ & 62,7 & 49,4 & 5,73 & $<0,001$ & 1,6 \\
\hline
\end{tabular}


Integrated care for the mentally deranged - Interfacial experiences Due to the separation of the outpatient and in-patient/partly in-patient means of treatment in Germany, our health care system is complicated even though the legislation provided by $\S 73$ German Social Code (SGB V, $\S 140$ a ff) has recently offered an opportunity to apply the so-called "integrated care". Additional alternatives are provided for by legislation ( $§ 73$ SGB V) which could be applied in the psychiatric/psychotherapeutic sector but are not yet fully made use of. Treatment in an outpatient set-up could be effected more efficiently and hence more cost-effectively by cross-linking the therapists/medics/medical staff of different sectors and professions. A first step has been made with the help of the Netz für seelische Gesundheit in Mainz, Germany (Network for Mental Health). In this setup GPs, psychiatrists, psychotherapists, a German welfare association sponsoring a day clinic and a psychiatric mobile clinic as well as other medical practitioners have joined. Other bodies and institutions will link up. However, such co-operative initiatives lack financial support and are thus handicapped, rendering the German health care system more cumbersome than necessary.

Key words

Outpatient psychiatric care - networking - German health care system integrated care - reinstatement

\section{Danksagung}

Die statistische Auswertung erfolgte dankenswerterweise durch Herrn Dipl.Psych. Manfred Kießling, Psychologischer Psychotherapeut, Vorstandsmitglied des Netzes für seelische Gesundheit Mainz e. V.
- SCL-90-R, eine Symptomcheckliste zur Messung der subjektiv empfundenen Beeinträchtigung durch körperliche und psychische Symptome innerhalb eines Zeitraumes von 7 Tagen (darunter Somatisierung, Depressivität, Ängstlichkeit, paranoides Denken)

- SDS, die Self-rating depression scale, ein Selbstbeurteilungsfragebogen zur Depression

- GAF, den Global Assessment of Functioning, zur Beurteilung des allgemeinen Funktionsniveaus

- VEV, der Veränderungsfragebogen des Erlebens und Verhaltens, zur Untersuchung von Veränderungen durch Psychotherapie.

\section{Evaluation}

Zum Zeitpunkt der Berichterstellung hatten abschließend 22 Patienten am IV-Projekt teilgenommen. Von den Teilnehmern waren 15 Frauen und 7 Männer in einem Durchschnittsalter von 45,7 bei einer Spannweite von $18-81$ Jahren. In 19 Fällen war die Hauptdiagnose Depression. Die Teilnehmer äußerten sich ausgesprochen zufrieden über das Projekt und gaben hochsignifikante positive Veränderungen in ihrem Erleben an. Für die Evaluation des Projekts wurden die Teilnehmer gebeten zu Beginn und zum Ende der Behandlung im Rahmen einer Integrierten Versorgungsbehandlung die Symptomcheckliste SCL-90-R von Derogatis (2) auszufüllen. Desweiteren wurden diagnosespezifische Tests in die Untersuchung miteinbezogen, auf die hier nicht näher eingegangen werden soll, da bisher zu wenig Daten für diese Tests vorliegen. Der SCl-90-R wurde bisher von 13 Projektteilnehmern beantwortet. Die 9 Einzelskalen und die 3 globalen Kennwerte wurden einem statistischen t-Test für abhängige Messungen unterzogen. Zusätzlich wurden die Effektstärken nach Cohen (1) berechnet. Die Ergebnisse sind in Tab. 1 zusammengefasst.

Alle t-Tests führen zu signifikanten Ergebnissen für die 2-seitige Fragestellung. Lediglich bei den Skalen ,Ängstlichkeit' und ,Aggressivität' liegt das Signifikanzniveau zwischen 5 und $1 \%$. Bei 7 Testungen wird das Signifikanzniveau von 0,001 unterschritten. Für die Effektstärken finden sich dementsprechend bei den Skalen Ängstlichkeit und Aggressivität auch hier die schwächsten Ergebnisse, dennoch wird eine mittlere Effektstärke zwischen 0,5 und 0,8 ermittelt. Alle anderen Effektstärken sind hoch.

Die vorliegende Evaluation entspricht nicht höheren wissenschaftlichen Anforderungen mit z. B. Vergleichen mit Kontrollgruppen oder doppelblinden Versuchsplänen. Auch sollten die Effektstärken alleine nicht überinterpretiert werden. In Kombination mit den durchweg signifikanten Ergebnissen in den t-Tests jedoch interpretieren wir die Verbesserungen in den Testwerten als einen Hinweis auf deutliche Behandlungserfolge, was von den Patienten subjektiv ebenso beschrieben wird. Beim GAF-Test (Abb. 2) zeigte sich außerdem eine deutliche Verbesserung des Funktionsniveaus der Patienten durch die integrierte Behandlung.

\section{Fazit}

Ob dieses Projekt der Integrierten Versorgung herkömmlichen Behandlungspfaden gegenüber überlegen ist, lässt sich mit dieser Evaluation nicht beantworten. Dazu wäre weitere Forschungsarbeit notwendig. Die vorliegenden Ergebnisse lassen eine solche Vermutung aber durchaus zu.

Alles in allem ist durch die integrierte Versorgung ein deutlicher Schritt auf eine verbesserte Behandlung psychisch Kranker gemacht worden. Weitere Schritte sind angedacht.

\section{Literatur}

1 Cohen J. Statistical Power Analysis for the Behavioral Sciences. 2. Aufl. Hillsdale: Lawrence Erlbaum Associates, 1988

2 Franke GH. SCL-90-R, Symptom-Checkliste von L.R. Derogatis - Deutsche Version. 2. Aufl. Göttingen: Beltz Test; 2002

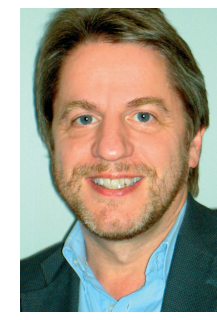

Korrespondenz

Dr. Siegfried Stephan

Vorsitzender des Netzes für seelische Gesundheit Mainz e. V. www.nsg-mainz.de 